\title{
The Detection of UHECRs with the EUSO-TA Telescope
}

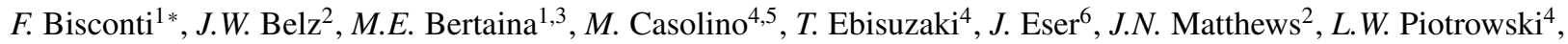 \\ Z. Plebaniak ${ }^{7}, H$. Sagawa $^{8}, N$. Sakaki $^{4}, H$. Shin $^{8}, K$. Shinozaki ${ }^{1,3}, P$. Sokolsky ${ }^{2}, Y$. Takizawa ${ }^{4}, Y$. Tameda ${ }^{9}$, and \\ G.B. Thomson ${ }^{2}$ for the JEM-EUSO Collaboration \\ ${ }^{1}$ Istituto Nazionale di Fisica Nucleare - Sezione di Torino, Turin, Italy \\ ${ }^{2}$ University of Utah, Salt Lake City, USA \\ ${ }^{3}$ Università di Torino, Turin, Italy \\ ${ }^{4}$ RIKEN, Wako, Japan \\ ${ }^{5}$ Istituto Nazionale di Fisica Nucleare - Sezione di Roma Tor Vergata, Rome, Italy \\ ${ }^{6}$ Colorado School of Mines, Golden, USA \\ ${ }^{7}$ National Centre for Nuclear Research, Lodz, Poland \\ ${ }^{8}$ Institute for Cosmic Ray Research, University of Tokyo, Kashiwa, Japan \\ ${ }^{9}$ Department of Engineering Science, Faculty of Engineering, Osaka Electro-Communication University, Neyagawa-shi, Osaka, Japan
}

\begin{abstract}
EUSO-TA is a cosmic ray detector developed by the JEM-EUSO (Joint Experiment Missions for Extreme Universe Space Observatory) Collaboration, observing during nighttime the fluorescence light emitted along the path of extensive air showers in the atmosphere. It is installed at the Telescope Array site in Utah, USA, in front of the fluorescence detector station at Black Rock Mesa. It serves as a ground-based pathfinder experiment for future space-based missions. EUSO-TA has an optical system with two Fresnel lenses and a focal surface with $6 \times 6$ multi-anode photomultiplier tubes with 64 channels each, for a total of 2304 channels. The overall field of view is $\sim 10.6^{\circ} \times 10.6^{\circ}$. This detector technology allows the detection of cosmic ray events with high spatial resolution, having each channel a field of view of about $\sim 0.2^{\circ} \times 0.2^{\circ}$ and a temporal resolution of $2.5 \mu \mathrm{s}$. First observations of ultra-high energy cosmic rays revealed the cosmic ray detection capability of EUSO-TA. The foreseen upgrade of EUSO-TA will improve the efficiency of the detector and will increase the statistics of detected events. In this work we present recent results of the detection capability of EUSO-TA and its limits. Moreover, other results about the analysis of laser pulses, stars and meteors will be discussed.
\end{abstract}

\section{Introduction}

EUSO-TA [1] is an experiment of the JEM-EUSO program [2], whose aim is to detect, from space, the rare ultra-high energy cosmic rays (UHECRs), with energy $\sim 10^{20} \mathrm{eV}$ and flux $\sim 1$ particle $/ \mathrm{km}^{2}$ per millennium. The detection of UHECRs is possible observing the UV fluorescence light emitted by Nitrogen molecules when extensive air showers induced by UHECRs (hereafter "showers") traverse the atmosphere used as a huge calorimeter. The probability to detect UHECRs is strongly increased from space with respect to ground, since the observed area projected on ground would be up to $\sim 10^{5} \mathrm{~km}^{2}$, much larger than any extension of a ground-based experiment.

The purpose of the EUSO-TA experiment is to validate the observation principle and the design of the detector by observing showers and laser pulses from ground. The location of EUSO-TA is strategic. Indeed, it is installed at the Telescope Array (TA) [3] site in Utah (USA), in front of the Black Rock Mesa fluorescence detector (BRM-FD) station [4]. From there, it is possible to detect showers in coincidence with BRM-FDs and pulsed laser shots from the Central Laser Facility (CLF) [5] can be used to test

*e-mail: francesca.bisconti@to.infn.it and calibrate the detector. In addition, portable lasers with variable direction and energy, like the Global Light System prototype (GLS) [6], are used to extend the range of distance of the source from the detector. The observation of shower and laser events will be discussed in this work, as well as the observation of stars and meteors, to understand the performance of EUSO-TA and its detection limit.

\section{The EUSO-TA detector}

\subsection{Detector design and acquisition mode}

EUSO-TA consists of an optical system with two flat Fresnel lenses sized $1 \mathrm{~m} \times 1 \mathrm{~m}$ and $8 \mathrm{~mm}$ thick [7]. The EUSO-TA focal surface has a concave shape. It consists of one photo-detector module (PDM), which is a $17 \mathrm{~cm} \times 17 \mathrm{~cm}$ active surface composed by an array of $6 \times 6$ Hamamatsu multi-anode photomultiplier tubes (MAPMTs, model R11265-M64) [8]. Each MAPMT has $8 \times 8$ pixels with side $2.88 \mathrm{~mm}$ for a total of 2304 pixels over the whole PDM, with a field of view of $0.2^{\circ} \times 0.2^{\circ}$ each. Each pixel has a gain (electron multiplication ratio) of more than $10^{6}$, which allows single photon counting 
and a photon detection efficiency of $\sim 30 \%$. A UV transmitting band pass filter in the range $290-430 \mathrm{~nm}$ is glued on top of each MAPMT. The field of view of EUSO-TA is $\sim 10.6^{\circ} \times 10.6^{\circ}$.

Data are sampled in gate time units (GTUs) corresponding to $2.5 \mu \mathrm{s}$ and representing the time resolution of the detectors within the JEM-EUSO program. This has been designed for the data acquisition of showers from space, corresponding to the maximum time for light to cross one pixel of MAPMT from $400 \mathrm{~km}$ altitude. The readout is performed by one 64-channel SPACIROC1 ASIC chip [9] per MAPMT, with a dead time at beginning of each GTU of $0.2 \mu \mathrm{s}$ and $30 \mathrm{~ns}$ double pulse resolution.

Like all fluorescence detectors, EUSO-TA works at nighttime and in the best cases in clear sky conditions, to reduce effects of atmospheric and cloud attenuation. The elevation of the instrument can be manually changed from $0^{\circ}$ to $30^{\circ}$ with respect to the horizon, whereas the azimuth is fixed at $53^{\circ}$ from North counterclockwise, pointing to the CLF.

Data are acquired in coincidence with BRM-FDs as external trigger: in case of a trigger signal received by BRM-FDs, a packet of 128 GTUs centered around the TA trigger is saved, which otherwise would be overwritten. This acquisition mode allows to know in advance if EUSO-TA could have detected showers, thanks to the large field of view of BRM-FDs which encompasses the EUSO-TA ones. Moreover, from the shower reconstruction made by the TA experiment, the nature of the event (i.e. arrival direction, impact point, energy etc.) is known and used for further analyses. However, since the field of view of TA is about 30 times that of EUSO-TA, the amount of data saved is larger than the actual data containing events, that makes the search of real events challenging.

\subsection{Upgrade of the detector}

EUSO-TA provides an excellent opportunity to test technology for existing and future experiments within the JEM-EUSO framework, as it allows stable field observations for extended time periods. To intensify the data acquisitions, an upgrade of the detector is foreseen in 2019. This will allow remote automated operations, making the collection of data possible continuously over the year. Furthermore, the sensitivity of the experiment will be enhanced, as the electronics will be improved with SPACIROC3 ASIC chips [10], with dead time at beginning of each GTU of $0.05 \mu$ s (instead of $0.2 \mu$ s for the currently used chip) and 5 ns double pulse resolution (instead of $30 \mathrm{~ns}$ ). This will make the detector more efficient, in particular for the detection of close showers, which cross individual pixels in short timescales. Moreover, the experiment will also be upgraded with advanced self-triggering capabilities, replacing the current PDM data processing board with a new board based on system-on chip (Zynq XC7Z030 FPGA [11]), with more memory and more resources. It allows the implementation of data read-out on three timescales. Data samples with resolution of $2.5 \mu \mathrm{s}$ are saved for UHECR observations; data samples with resolutions of $320 \mu \mathrm{s}$ (128 time frames of $2.5 \mu \mathrm{s}$ ) and $40.96 \mathrm{~ms}$ (128 time frames of $320 \mu \mathrm{s}$ ) are stored for slow events such as strangelets and meteors, detectable offline with dedicated event search algorithms.

\section{Observation of UHECR events}

In the year 2015 four data acquisition campaigns were done in coincidence with BRM-FDs, and one in 2016, for a total of about 140 hours (120 hours in 2015, to which this work refers).

Nine events have been detected. The information on the showers crossing the field of view of EUSO-TA, whether it detected them or not, allows to estimate the detection limit. TA observes these showers, since it has a field of view that includes the EUSO-TA ones, as visible in Figure 1(b). The TA Collaboration provides the list of such events and also the reconstructed parameters of the showers using the BRM-FDs data, i.e. in monocular mode: the shortest distance between the shower axis and the detector in the shower-detector plane, $R_{p}$; the reconstructed energy of the primary particle, $E_{\text {rectA }}$; and the impact point on ground of each shower axis. These parameters are essential to analyse and simulate the UHECR events. Analysis of the events will be discussed in the next sections. Simulations well reproduce the data, and details on this can be found in [12].

Figure 1 shows in (a) one of the UHECR events detected by EUSO-TA, with the counts per pixel on the full PDM, and in (b) the same event detected by BRM-FDs, in horizontal coordinates, with the EUSO-TA field of view indicated by the red rectangle and each circle representing one PMT of the BRM-FDs. This event has $R_{p}=2.5 \mathrm{~km}$ and $E_{\text {recTA }}=10^{18} \mathrm{eV}$, and lasts $1 \mathrm{GTU}$. One can see that the spatial resolution is higher for EUSO-TA than for the BRM-FDs. As the field of view of BRM-FDs covers $33^{\circ}$ in elevation and $100^{\circ}$ in azimuth, the event reconstruction is based on the observation of the whole or most of the shower, including the shower maximum, i.e. the point along the shower axis with the maximum number of particles. As previously mentioned, the reconstruction of the events provides the energy of the primary particle $E_{\text {recTA }}$. On the other hand, EUSO-TA has a field of view of $10.6^{\circ}$, so it observes just a small portion of the shower and in most cases it does not observe the shower maximum. In this way, EUSO-TA observes a part of the shower that suggests less energy in the electromagnetic component than it actually has when observing the full shower. Therefore, to understand the detection capability of EUSO-TA, it is necessary to consider such equivalent energy $E_{e q}$ instead of $E_{\text {recTA }}$. With respect to what discussed in reference [1], here an update on the analysis towards understanding the detection limit of EUSO-TA is described.

First, the minimum distance between the shower axis and EUSO-TA in the shower-detector plane, i.e. the impact parameter $R_{p}$, was considered in the previous analysis. However, this distance in most of the cases does not correspond to the distance that photons from the shower travel to the detector, in particular for inclined showers with small impact parameter. Therefore, the distance of 


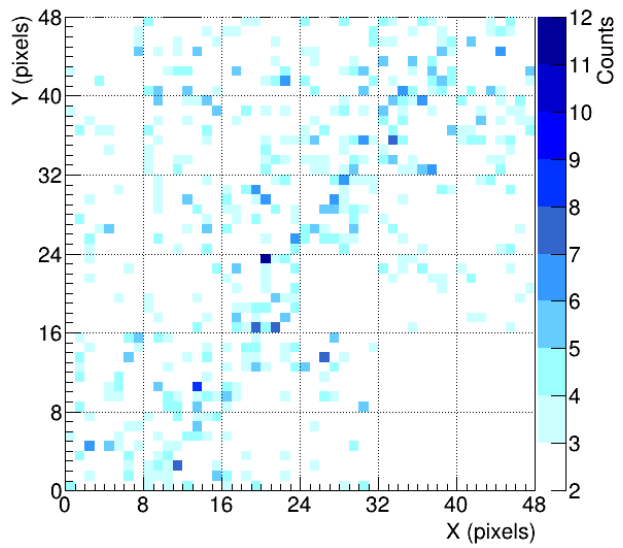

(a) UHECR event detected by EUSO-TA with counts per pixel on the full PDM on 1 GTU, i.e. $2.5 \mu \mathrm{s}$.

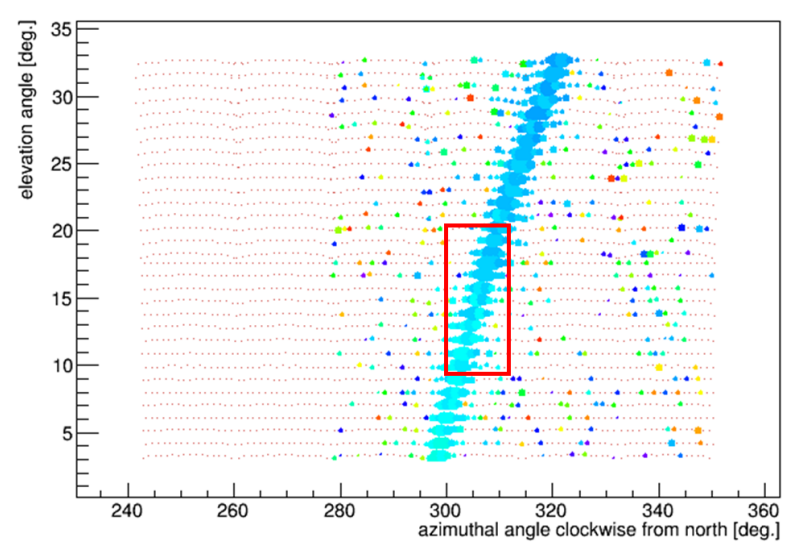

(b) UHECR event detected by BRM-FD. The marker size is proportional to the number of photons arriving on a PMT and the color corresponds to the arrival time. The signal is integrated within a $51.2 \mathrm{~ms}$ time window. Image by courtesy of the TA Collaboration. The red rectangle shows the EUSO-TA field of view.

Figure 1: The same UHECR event detected by EUSOTA (a) and BRM-FDs (b) on May 13th, 2015, with $R_{p}=$ $2.5 \mathrm{~km}$ and $E_{\text {recTA }}=10^{18} \mathrm{eV}$.

the shower axis along the optical axis of the detector, with a certain elevation, is used in this updated analysis.

Second, to calculate $E_{e q}$ for the showers that crossed the EUSO-TA field of view, a conversion factor is calculated in the following way and then multiplied by $E_{\text {recTA }}$. Given the direction (zenith and azimuth angles) and the energy of a shower, simulations with CONEX [13] provide the distribution of the number of photons with respect to the altitude in the atmosphere. From the direction of the shower, its impact point on ground and the elevation angle that EUSO-TA had when the shower crossed its field of view, it is possible to calculate the altitude at which EUSO-TA observed (or did not) such shower. The conversion factor is the ratio between the number of photons arriving at the detector with the actual elevation, and the number of photons in the case the detector was pointing to the shower maximum. In the previous analysis, the integral of the distribution of the number of photons within the field of view was considered. However, the distance of photons traveling from the same shower within the field of view may vary significantly, in particular for inclined showers with a small impact parameter. To reduce the systematic uncertainties introduced by this effect, just the number of photons emitted at the point where the distance between the telescope's optical axis and the shower axis is minimum is considered when calculating the energy conversion factor. This can be further revised in near future, when also the atmospheric attenuation will be considered, together with other possible minor corrections.

Figure 2(a) shows the showers which crossed the EUSO-TA field of view with respect to their $E_{\text {recTA }}$ and $R_{p}$, where red crosses represent the non-detected events and green stars represent the detected events. A separation between detected and non-detected events is already visible, as the detected events are with higher energy and lower impact parameter. Figure 2(b) shows the same events, but plotted with their equivalent energy $E_{e q}$ and the distance along the telescope axis to the shower. Here the separation between non-detected and detected events is more visible. As the equivalent energy of the shower is usually lower than the reconstructed energy, all points moved to lower energies with respect to the former plot. Moreover, since the distance of the shower along the telescope axis is usually longer than the impact parameter, all the points moved towards longer distances. An analytical fit of the upper detected events (highlighted with black circles) was made with a second order polinomial. All the detected events are supposed to be below and on the right of the line. A few non-detected events also appear in this region. This can be connected to the dead time of $0.2 \mu$ s the electronics has at the beginning of the GTUs, which makes it possible that the detector misses some events. In addition, laser events from the CLF and the GLS (with two energies), are plotted with triangles and squares, respectively. As the laser events are clearly visible above the night sky background, they stay well away on the right side of the line.

\section{Other results}

EUSO-TA was mainly designed for the detection of UHECRs and laser beams in the UV band, where the laser is useful for the calibration of the detector. However, it revealed to be capable of detecting other kind of light sources like stars and meteors, which can initiate studies regarding the performance of the detector and phenomena in wavelength bands and timescales not investigated yet from other experiments. Results presented hereafter refer to reference [1].

\subsection{Lasers}

In order to study the response of EUSO-TA to a known light source, laser pulses from TA's CLF [5], located at about $21 \mathrm{~km}$ in front of EUSO-TA and BRM-FD station, have been used. The CLF shoots a laser pulse of $355 \mathrm{~nm}$ wavelength vertically. During standard observations, laser pulses are shot every half an hour for $30 \mathrm{~s}$ at a shooting frequency of $10 \mathrm{~Hz}$. The scattered light of the $3 \mathrm{~mJ}$ beam is clearly visible when traversing the EUSO-TA field of 


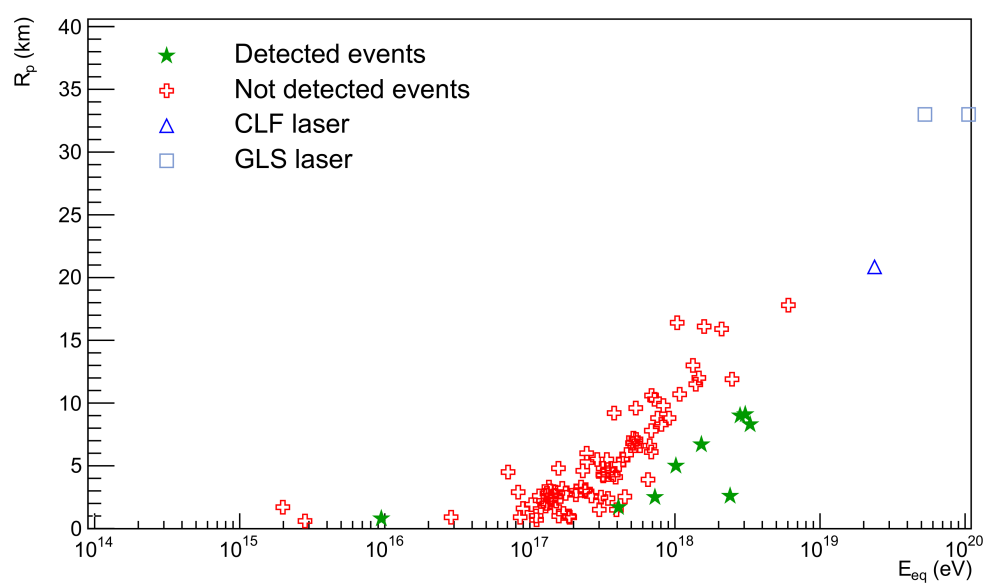

(a) Events crossing the EUSO-TA field of view with their impact parameter $R_{p}$ and reconstructed energy $E_{\text {recTA }}$

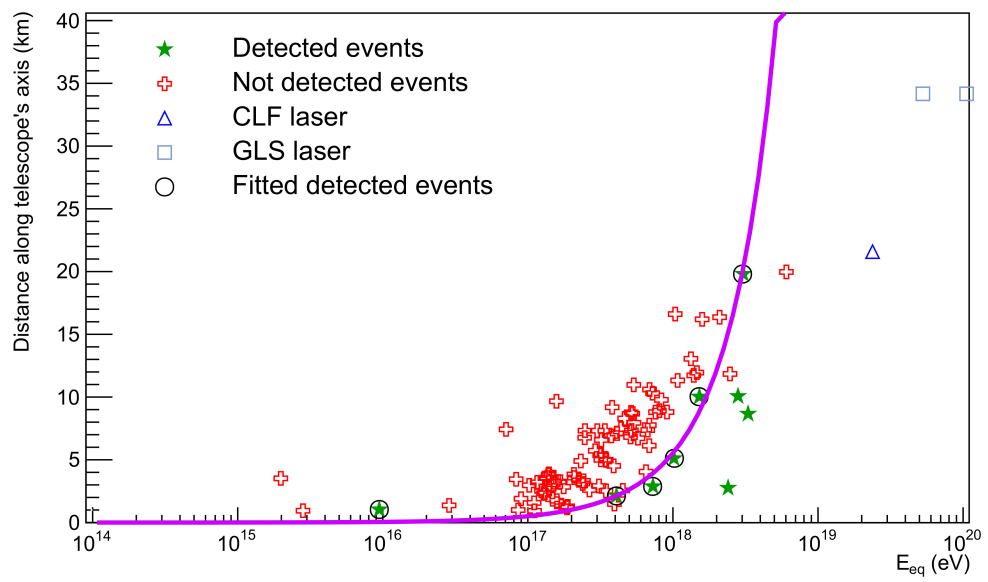

(b) Events crossing the EUSO-TA field of view with their distance along the telescope axis and calculated equivalent energy $E_{e q}$. The line shows a fit of the "upper" detected events with a second order polynomial.

Figure 2: Showers induced by UHECRs crossing the field of view of EUSO-TA before (a) and after (b) the conversion to the equivalent energy and the calculation of the distance along the telescope axis.

view. A laser event lasts 6 to 8 GTUs, i.e. it is visible as a moving spot on 6 to 8 consecutive data frames. This depends on the shot and acquisition time synchronization and also on the elevation angle of the EUSO-TA axis during the observation. For higher elevation angles the vertical path of the laser pulse in the field of view is longer than for low elevation angles. The spot length is $6-8$ pixels, depending on the position on the frame (see Figure 3(a), inset), which is consistent with expectations. The width of the CLF track changes depending on the position with respect to the center, from about 3.8 pixels at the edges of the field of view to about 2.8 pixels in the center, as visible in Figure 3(a), showing the average of 259 tracks of the CLF laser. In the central part, the laser track is less affected by aberrations, and the width is consistent with the measured point spread function of star images, whose details will be reported later on.

In addition to the CLF measurements, observations of laser beams from the GLS [6], a mobile UV laser device, were also performed. The laser can shoot with energies in the range of about $1-86 \mathrm{~mJ}$, with the pointing direction adjustable in two dimensions. Initial results of the direction reconstruction are shown in Figure 3(b). The detection limit for the laser shots of energies $2-4 \mathrm{~mJ}$ implies an energy threshold for detectable UHECR of energies in the range $10^{19.7}-10^{20} \mathrm{eV}$ at $33 \mathrm{~km}$ distance [14].

\subsection{Stars}

Stars can be used as point sources to analyze the sensitivity, the dimension of the field of view and the point spread function of the detector. EUSO-TA can observe stars with apparent magnitude in the B-band up to $m_{B} \simeq 5.5$ on sums of 1280 frames (about $3.2 \mathrm{~ms}$ integration time). This limiting magnitude is a very approximate measure of the EUSO-TA sensitivity, since it depends largely on the spectral properties of the star in question and atmospheric conditions at the time of observation. Moreover, a small number of sufficiently bright stars in the field of view do not allow for a precise estimate. Frame stacking can be used 


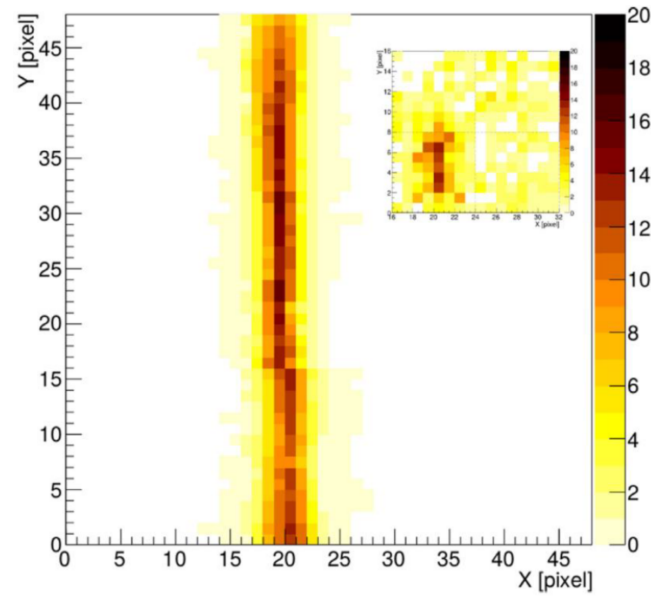

(a) Average counts of 259 tracks of the CLF laser shoots The inset shows a part of a single frame containing a laser pulse.

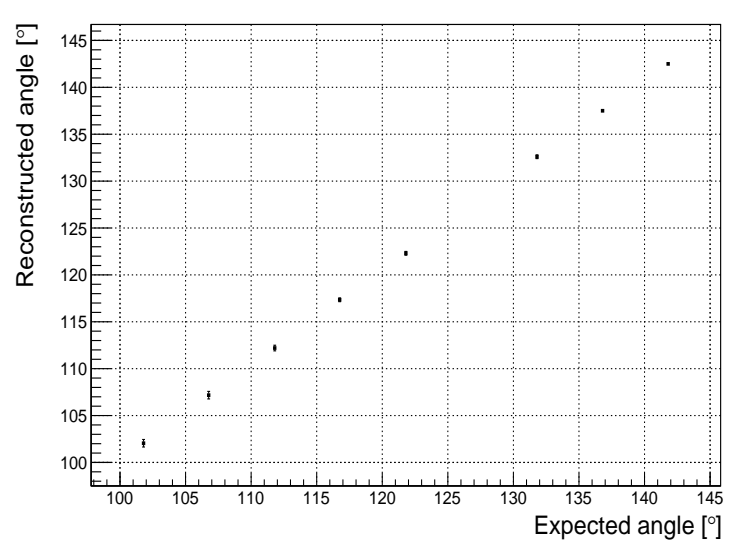

(b) Reconstructed angle with respect to expected angle for GLS laser pointing sweep. Error bars show the standard deviation of the distribution of reconstructed angles.

Figure 3: Plots related to the analysis of the laser shoots from the CLF (a) and GLS (b).

to achieve a better signal to noise ratio, thanks to negligible movements of the stars on the sky for short observation timescales compared to the angular size of a pixel. An example of 1280 stacked frames with a few stars clearly visible is shown in Figure 4. The brightest star positions are marked on the image following the Hipparcos catalog [15]. The very wide field of view results in an asymmetric point spread function in regions of the focal surface far from the optical axis due to aberrations such as coma or astigmatism. An approach making use of an elliptical Gaussian profile fit gives an asymmetric point spread function with an average full width at half maximum of 2.98 and 2.40 pixels for the major and minor axis, respectively [16]. This result is well within the requirements for observations of showers.

\subsection{Slow events}

Although EUSO-TA is designed for observations of $\mu \mathrm{s}$ scale events, it can also monitor phenomena taking place

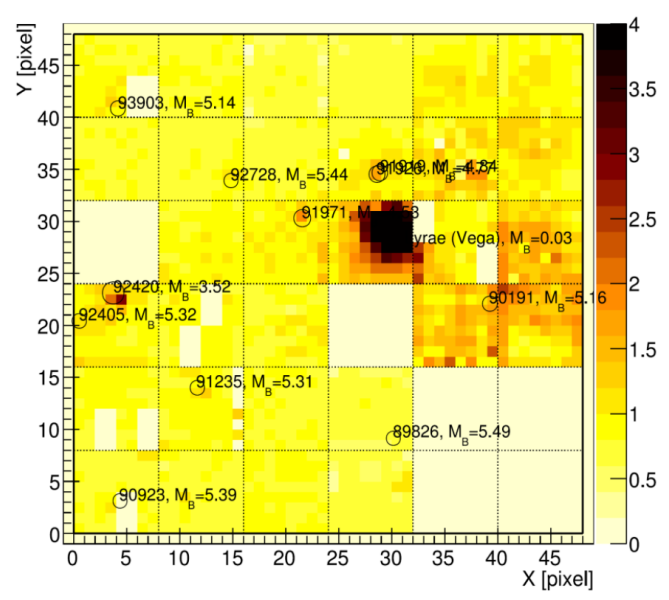

Figure 4: Stars imaged by the sum of counts of 1280 data frames ( 3.2 ms observation time). Stars are labeled with the Hipparcos catalogue number or name (for the most brilliant) and with their apparent magnitudes in the B-band up to 5.5 .

on much longer timescales. The most common are flashes from airplanes and satellites reflecting sunlight. Satellite observations can be used as a proof of concept for the plans of space debris remediation with future, orbital JEMEUSO-like experiments [17].

EUSO-TA has also observed meteors. This capability allows to study the details of the ablation processes undergone by the meteoroid through the atmosphere with higher time resolution $(\sim \mu$ s to $\sim s)$ than other detectors designed for meteor observations. High time resolution and large field of view are important also for the detection of a phenomenon in appearance similar to meteors, i.e. strangelets [18]. They are made of strange matter and predicted to interact with the atmosphere producing light, but with a different evolution compared to meteors due to the fact that they do not fragment or lose mass. The faintest meteor observed had an apparent magnitude of $m_{B}=2.4 \pm 0.1$, which was bright enough to detect. If the same detection limit of $m_{B}=5.5$ as for stars is assumed, then an average detection rate of about 1 meteor/hour of observation is expected, upon the introduction of a dedicated trigger algorithm. To set limits on strangelets more stringent than existing limits [18], a dedicated autonomous trigger is in preparation, as the external trigger from BRM-FDs is designed to discard stationary or slow moving events.

\section{Summary and Outlook}

EUSO-TA demonstrates the performance of a new detector technology for the observation of UHECRs, based on Fresnel lenses and MAPMTs. The detector has registered, using BRM-FD triggers, nine UHECRs events during its five observational campaigns. The response of the detector was tested using UV laser shots mimicking extensive air showers. Additionally, a number of "slow" events such as stars, meteors and airplanes have been observed, that extended the scientific objectives. The nine registered 


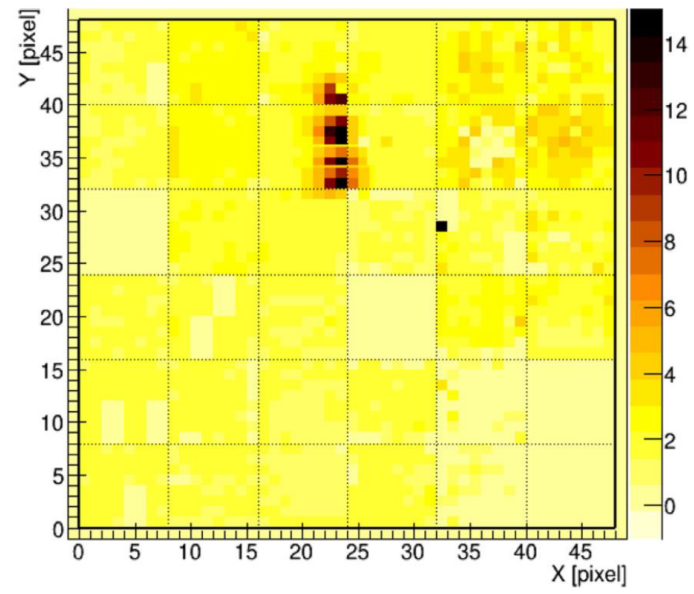

Figure 5: Meteor track detected on the night November 12th, 2015. Overlap of four averages of 1280 frames (12.8 ms total integration time). The apparent magnitude varied from 2.4 to 4.1

UHECRs gave hints on the EUSO-TA sensitivity, which is further studied. The main goal of EUSO-TA to test the capabilities and stability of the hardware proved an invaluable testbench for the modifications applied in the next generation experiments within the JEM-EUSO program. Moreover, an optimization of the detector will be achieved through the hardware upgrades in the near future.

\section{Acknowledgments}

The support received by the Telescope Array Collaboration is deeply acknowledged. This work was partially supported by Basic Science Interdisciplinary Research Projects of RIKEN and JSPS KAKENHI Grant (22340063, 23340081, and 24244042), by the Italian Ministry of Foreign Affairs and International Cooperation, by the Italian Space Agency through the ASI INFN agreement n. 2017-8-H.0, by NASA award 11-APRA0058 in the USA, by the French space agency CNES, by the Deutsches Zentrum für Luft- und Raumfahrt, the Helmholtz Alliance for Astroparticle Physics funded by the Initiative and Networking Fund of the Helmholtz Association (Germany), by Slovak Academy of Sciences MVTS JEM-EUSO, by National Science Centre in Poland grant (2015/19/N/ST9/03708), by Mexican funding agencies PAPIIT-UNAM, CONACyT and the Mexican Space Agency (AEM), as well as VEGA grant agency project 2/0132/17, and by State Space Corporation ROSCOSMOS and Russian Foundation for Basic Research (grant 16-2913065).

\section{References}

[1] G. Abdellaoui et al. (JEM-EUSO Coll.), "EUSO-TA - first results from a ground-based EUSO telescope",
Astropart. Phys. 102, 98-111 (2018)

[2] J.H. Adams Jr. et al. (JEM-EUSO Coll.), “JEM-EUSO observational technique and exposure", Exp. Astron., 40, 117-134 (2015)

[3] M. Fukushima et al., "Telescope Array project for extremely high energy cosmic rays", Prog. Theor. Phys. Suppl., 151, 206-210 (2003)

[4] H. Tokuno et al., "New air fluorescence detectors employed in the Telescope Array experiment", Nucl. Instrum. Meth. A, 676, 54-65 (2012)

[5] Y. Takahashi et al., "Central laser facility analysis at the Telescope Array experiment", AIP Conference Proc., 1367, 157-160 (2011)

[6] P. Hunt et al. (JEM-EUSO Coll.), "The JEMEUSO global light system laser station prototype", PoS(ICRC2015)626 (2016)

[7] Y. Hachisu et al., "Manufacturing of the TA-EUSO and the EUSO-Balloon lenses", Proc. of ICRC2013, ID1040 (2013)

[8] H. Prieto-Alfonso et al. (JEM-EUSO Coll.), "Multi anode photomultiplier tube reliability assessment for the JEM-EUSO space mission", arXiv:1501.05908 [physics.ins-det] (2015)

[9] H. Miyamoto et al. (JEM-EUSO Coll.), "Performance of the SPACIROC front-end ASIC for JEM-EUSO", Proc. of ICRC2013, ID1089 (2013)

[10] S. Blin-Bondil, et al., "SPACIROC3: A Front-End Readout ASIC for JEM-EUSO cosmic ray observatory". Proc. of TIPP 2014, PoS(TIPP2014)172 (2014).

[11] Xilinx. Zynq-7000 All Programmable SoC (2016) URL: https://www.xilinx.com/products/silicondevices/soc/zynq-7000.html

[12] F. Bisconti et al. (JEM-EUSO Coll.), "Simulation study of the detected and expected events for the EUSO-TA fluorescence detector", PoS(ICRC2017)463 (2017)

[13] T. Bergmann et al., "One-dimensional hybrid approach to extensive air shower simulation", Astropart. Phys., 26 420-432 (2007)

[14] A.L. Cummings, "Field Testing for EUSO-SPB: Logistics and First Results", master thesis, Colorado School of Mines. Arthur Lakes Library (2017)

[15] M.A. Perryman et al., "The HIPPARCOS catalogue”, Astron. Astrophys. 323 L49-L52 (1997)

[16] Z. Plebaniak et al. (JEM-EUSO Coll.), "Point Spread Function of EUSO-TA detector", PoS(ICRC2017)460 (2017)

[17] T. Ebisuzaki et al., "Demonstration designs for the remediation of space debris from the International Space Station”, Acta Astronaut. 112, 102-113 (2015)

[18] J.H. Adams Jr. et al., "JEM-EUSO: Meteor and nuclearite observations”, Exp. Astron. 40, 253-279 (2015) 